\title{
Th17 cells are associated with the Th1/Th2-cell balance during Echinococcus multilocularis infection
}

\author{
XIUMIN MA ${ }^{1,2^{*}}$, LIANG WANG $^{1 *}$, HUI ZHAO $^{1}$, NANNAN PANG $^{1,2}$, FENGBO ZHANG $^{1,2}$, TAO JIANG $^{1}$, \\ XUELEI LIU ${ }^{1,2}$, WULAMU-MAMUTI ${ }^{1,2}$, HAO WEN $^{1}$ and JIANBING DING ${ }^{1,3}$
}

${ }^{1}$ State Key Laboratory Incubation Base of Xinjiang Major Diseases Research and Xinjiang Key Laboratory of Echinococcosis, First Affiliated Hospital of Xinjiang Medical University; ${ }^{2}$ College of Basic Medicine of Xinjiang Medical University;

${ }^{3}$ National Clinical Research Base of Traditional Chinese Medicine of Xinjiang Medical University,

Urumqi, Xinjiang 830011, P.R. China

Received August 23, 2013; Accepted February 19, 2014

DOI: $10.3892 / \mathrm{mmr} .2014 .2170$

\begin{abstract}
The present study investigated the immunopathological effect of Echinococcus multilocularis (Em) using cytokine detection. Expression of the T-helper (Th) 17-cytokine, interleukin 17 (IL-17), was observed using immunohistochemical staining, and levels of cytokines, including IL-17, transforming growth factor $\beta 1$ (TGF- $\beta 1$ ), IL-6, interferon $\gamma$ (IFN- $\gamma$ ) and IL-4, were assessed using ELISA at different stages of infection. IL-17 expression occurred in hepatic cells at 1 month post-infection, reached a maximum at 3 months post-infection and then decreased gradually. Compared with the uninfected control, levels of the cytokines IL-17, TGF- $\beta 1$, IL- 6 , IFN- $\gamma$ and IL-4 exhibited different dynamic patterns when infected with Em. In the immune response during the whole infection period, Th17 cells play an important role by secreting IL-17, which may be involved in the Th1/Th2-cell balance during the immune response. Th17 cells are associated with immunopathology in Em infection.
\end{abstract}

Correspondence to: Dr Jianbing Ding, National Clinical Research Base of Traditional Chinese Medicine of Xinjiang Medical University, No. 393 Xinyi Road, Urumqi, Xinjiang 830000, P.R. China E-mail: maxiumin1210@sohu.com

Professor Hao Wen, State Key Laboratory Incubation Base of Xinjiang Major Diseases Research and Xinjiang Key Laboratory of Echinococcosis, First Affiliated Hospital of Xinjiang Medical University, No. 137 Liyushan South Road, Urumqi, Xinjiang 830011, P.R. China

E-mail: dr.wenhao@163.com

*Contributed equally

Key words: Echinococcus multilocularis, interleukin 17, interleukin 6 , transforming growth factor- $\beta$

\section{Introduction}

Alveolar echinococcosis (AE) is a rare but fatal disease caused by infection with the larvae (metacestodes) of Echinococcus multilocularis $(\mathrm{Em})$. The disease has been fatal in $>95 \%$ of cases in the past 10 years. As a chronically progressive hepatic infestation, $\mathrm{AE}$ is characterised by a long asymptomatic period in which development of an invasive tumour-like, multi-vesiculated and exogenously budding lesion occurs (1).

In certain parasitic infection models, $\mathrm{T}$ helper $(\mathrm{Th})$ cells develop gradually to a certain polarised direction of a $\mathrm{T}$ subset, which participates in multiple pathological injuries and is able to cause tissue damage (2). With parasitic antigen stimulation, native $\mathrm{T}$ cells selectively differentiate into Th1 or Th2 cells $(3,4)$. Th1 cells play an important role in the defence against intracellular parasitic infections by secreting interleukin 2 (IL-2), interferon $\gamma($ IFN- $\gamma$ ) and tumour necrosis factor- $\beta$ (TNF- $\beta$ ), which mediate cytotoxic effects by promoting cytotoxic lymphocytes, natural killer cells and macrophages into activation and proliferation. Since the secreted TNF- $\beta$ and IFN- $\gamma$ can recruit and activate inflammatory cells, the Th1 immune response has often been associated with inflammation and tissue damage, resulting in delayed hypersensitivity. Th2 cells are involved in humoral immune responses and primarily provide efficient help for B-cell proliferation and antibody production. The Th1/Th2 imbalance is the most important immunological change in response to pathogenesis, and the immune response often gradually shifts towards a Th2 response at later stages of infection to prevent Th1-mediated damage (5).

Identified as a new subset of $\mathrm{T}$ helper cells not associated with Th1 or Th2 cells, Th17 cells are not only involved in the host defence against extracellular pathogens, but are also associated with the induction of autoimmunity and inflammatory responses (6). Previous studies have demonstrated that Th17 cells and IL-17 play an important role in parasitic schistosomiasis and toxoplasmosis (7-9); however, their role in $\mathrm{AE}$ has yet to be reported. In order to investigate the immunopathological regulation in Em infection, levels of different cytokines were assessed following infection with Em. The identification of the role of Th17 cells in AE development may be beneficial in explaining the cellular immunity observed in the Th1/Th2 axis. 

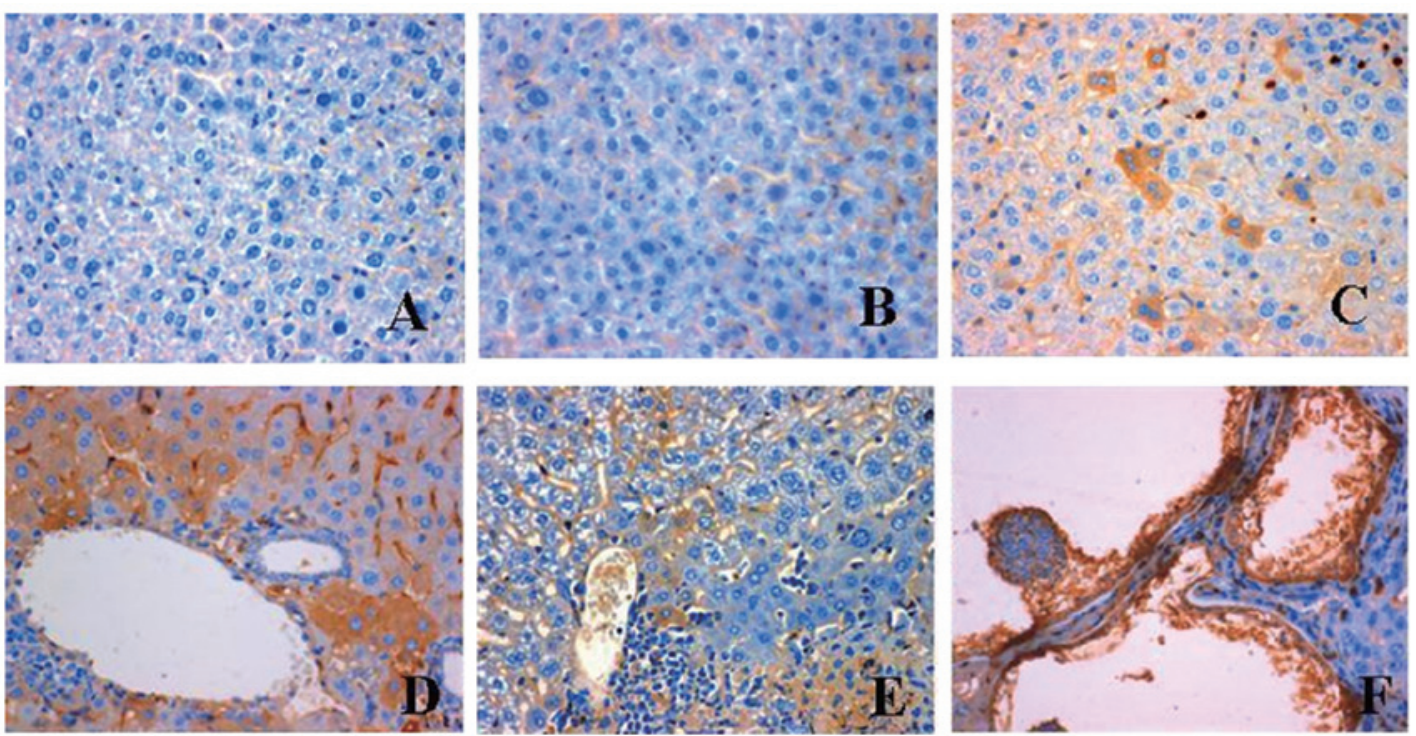

Figure 1. Expression of interleukin-17 in the livers of all infected groups (immunohistochemical staining; magnification, $\mathrm{x} 400$ ). (A) Uninfected control group; (B-F) groups at (B) 2 days, (C) 1 month, (D) 3 months, (E) 6 months and (F) 10 months post-infection.

\section{Materials and methods}

Animals and grouping. Twenty-four female BALB/c mice were provided by Xinjiang Medical University (Urumqi, China) and randomly divided into eight groups: piD2 (2 days post-infection, $n=3$ ), piD8 ( 8 days post-infection, $n=3$ ), piM1 ( 1 month post-infection, $\mathrm{n}=3$ ), piM2 ( 2 months post-infection, $\mathrm{n}=3$ ), piM3 (3 months post-infection, $\mathrm{n}=3$ ), piM6 (6 months post-infection, $n=3$ ), piM10 (10 months post-infection, $n=3$ ) and a control ( $n=3$, uninfected group). Experimental mice were infected by intraperitoneal injection of a metacestode suspension. Serum and liver samples were collected 2 days, 8 days and 1, 2, 3, 6 and 10 months post-infection, respectively. The care of the animals and all procedures were approved by the Institutional Animal Care and Use Committee, and mice were used in accordance with the ethical guidelines of the First Affiliated Hospital of Xinjiang Medical University.

Immunohistochemical staining. Formalin-fixed and paraffin-embedded liver biopsy specimens were cut into 4- $\mu \mathrm{m}$ sections and unwaxed in xylene. Following washing with ethanol several times, sections were stained with anti-IL-17 antibodies (Wuhan Boster Biological Technology, Ltd., Wuhan, China) at a dilution of 1:100.

ELISA. Serum levels of cytokines IL-17, TGF- $\beta 1$, IL-6, IFN- $\gamma$ and IL-4 were determined using ELISA. Cytokine assays were performed with ELISA kits (Ebioscience, San Diego, CA, USA) for each individual mouse, in accordance with the manufacturer's instructions.

Statistical analysis. All experiments were performed in triplicate. Data are expressed as the mean \pm standard deviation. One-way analysis of variance was applied to compare the differences among the different groups. A value of $\mathrm{P}<0.05$ was considered to indicate a significant difference between values.

\section{Results}

IL-17 expression in the livers of BALB/c mice infected with Em. To assess IL-17 expression in the livers of BALB/c mice infected with Em, immunohistochemical staining was performed. Typical pathological characteristics of the Em-infected mice included various vesicles with a diameter of $0.1 \mathrm{~mm}-3 \mathrm{~cm}$ and tumour-like growth in the liver with invasion of neighbouring tissue and organs. Staining for IL-17 expression was negative in the uninfected control sections (Fig. 1A). No expression of IL-17 was observed in the cytoplasm of hepatic cells at 2 days post-infection (Fig. 1B), while IL-17 was only expressed in a few hepatic sinusoid endothelial and hepatic cells at 1 month post-infection (Fig. 1C). Notably, at 3 months post-infection, a strong positive staining for IL-17 was found in hepatic and Kupffer cells, particularly in those around the central veins of the livers (Fig. 1D). At 4 months post-infection, parasitic germinal and laminated layers were formed in the liver, and the perisinusoidal spaces were enlarged. A few stained hepatic cells were observed at 6 months post-infection (Fig. 1E). At 10 months post-infection, the parasitic vesicles were surrounded by a necrotic zone and a large number of fibroblasts. Both the parasitic germinal layer and the hepatic cells surrounding the parasitic vesicles were intensively stained for IL-17 (Fig. 1F). In summary, IL-17 expression occurred in hepatic cells at 1 month post-infection, reached a maximum at 3 months post-infection and then decreased gradually.

Dynamic changes in cytokine levels in mice infected with Em. To assess the dynamic change in cytokine levels in mice infected with Em, ELISA was performed. The concentration of IL-17 remained low in uninfected controls. During the infection, a trend of constantly increasing IL-17 levels was observed, with levels at 6 and 10 months post-infection significantly higher than those in the control. Levels at 6 and 10 months post-infection were also significantly higher than those in the groups at 2 and 8 days post-infection, respectively (Fig. 2, Table I). 


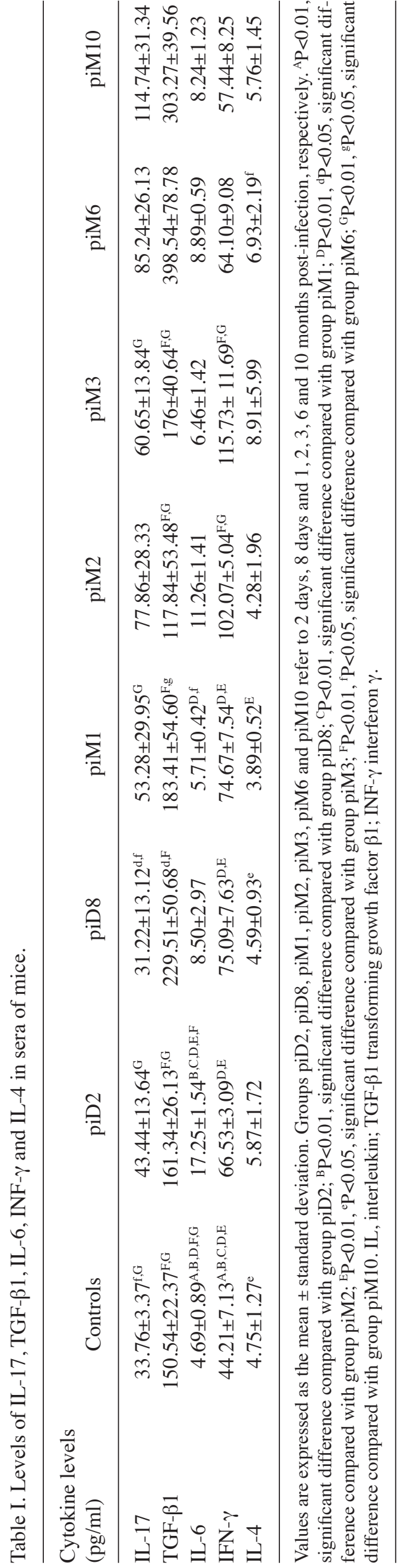

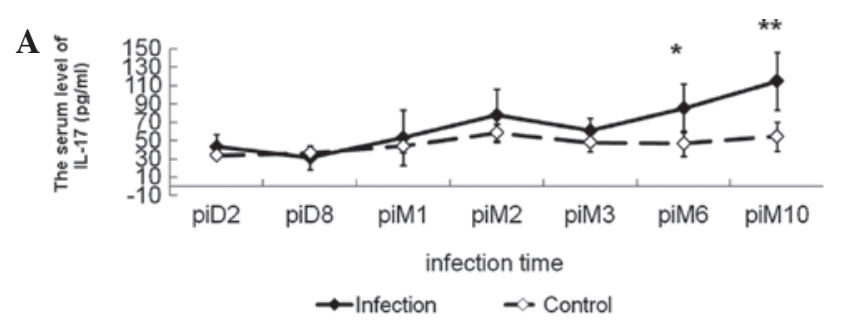
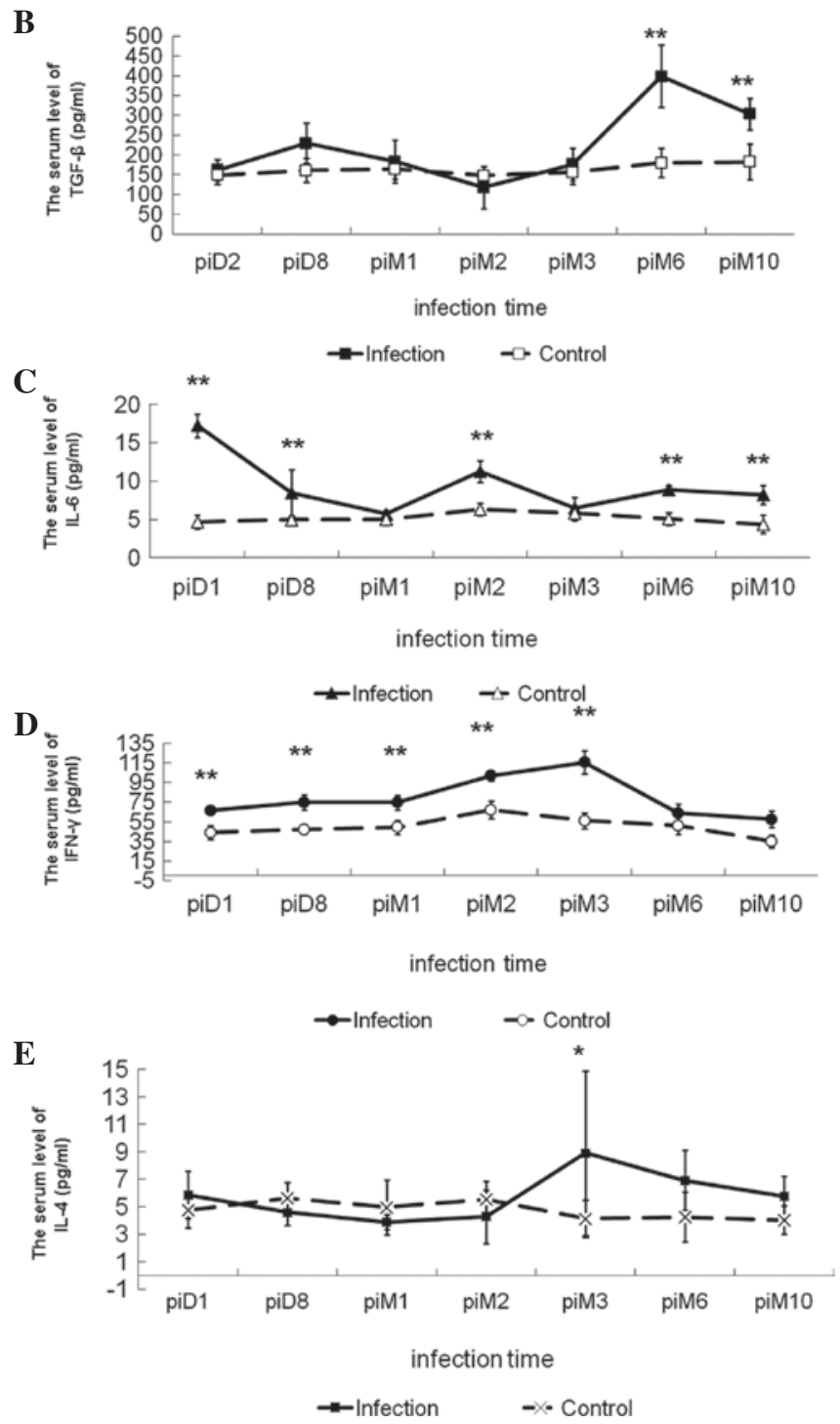

Figure 2. Changes in cytokine levels in serum of BALB/c mice infected with Echinococcus multilocularis. Levels of (A) IL-17, (B) TGF- $\beta 1$, (C) IL-6, (D) IFN- $\gamma$ and (E) IL-4. ${ }^{*} \mathrm{P}<0.05,{ }^{* *} \mathrm{P}<0.01$, significant difference compared with group control. IL, interleukin; TGF- $\beta$, transforming growth factor- $\beta$; IFN- $\gamma$, interferon- $\gamma$; pi, post-infection; D, day; M, month.

Expression of TGF- $\beta 1$ showed a similar trend to IL-17. Compared with the control and the groups at 2 days, 8 days and 1,2 and 3 months post-infection, the TGF- $\beta 1$ concentration was significantly increased at 6 and 10 months post-infection (Fig. 2, Table I). Levels of IL-6 reached a maximum at 2 days post-infection and then decreased to a minimum at 1 month post-infection, prior to steadily increasing until 2 months post-infection. IL- 6 production in all infected groups was significantly higher than that in the uninfected control (Fig. 2, Table I). IFN- $\gamma$ levels increased following Em infection, 
reaching a maximum at 3 months post-infection and decreasing thereafter, while the IL-4 levels remained low until 2 months post-infection and increased at 3 months post-infection (Fig. 2, Table I). These results suggest that Th17 cells may be involved in the Th1/Th2-cell balance during the immune response.

\section{Discussion}

A previous study suggested that IFN- $\gamma$ (Th1) activity was associated with the killing of both the protosceles and established cysts of E. granulosus (10). However, Wei et al (11) suggested that the induction of Th2-antibody-mediated immunity (AMI), with a parallel expansion of Th1-cell-mediated inflammatory (CMI) responses, was an important mechanism for host defence against metacestodes. Th1 CMI has an important role at the early stages of infection, while Th2 AMI is crucial for the later stages of infection.

A variety of immune mechanisms exist at an early stage of secondary E. granulosus infection, and Th cells show no dominant imbalance (12). It has been shown that during the intermediate stage of infection, when hydrated antigen levels in vivo are comparatively low, the immune response becomes Th2-skewed. On entering the chronic infection stage, the number of antigens gradually increases and Th1 cells become increasingly activated. This suggests that the early induction of Th2, with a parallel expansion of Th1, cells represents an important mechanism involved in the host defence against E. granulosus.

IFN- $\gamma$, induced by Th1-type antigens, can inhibit the secretion of Th2 cytokines in the anti-parasitic response primarily by enhancing macrophage functions and limiting the proliferation of intracellular parasitic protozoa in order to control the infection (13). By contrast, IL-4, produced by activated Th2 cells, stimulates the proliferation and differentiation of $\mathrm{B}$ cells to inhibit cell proliferation and the Th1 response. The present study showed that levels of IFN- $\gamma$ increased gradually following Em infection, reached a maximum at 3 months post-infection and then decreased. Levels of Th2-type cytokines were also high at 3 months post-infection. This suggests that the dominant Th1 immune response is shifted to a Th2 response in Em infection. In addition, IFN- $\gamma$ can promote Th1-cell maturation and inhibit Th2-cell function by reducing the production of Th2-type cytokines. Furthermore, macrophages activated by IFN- $\gamma$ can kill parasites and inhibit the growth of hydatid tissue. By contrast, high IL-4 levels delay the secretion of Th1-type cytokines and cell proliferation. In the present study, the levels of IL-4 remained low until 2 months post-infection, and increased after 3 months. As a result of the high secretion of IL-4, decreased levels of Th1-type cytokines led to rapid growth of Em tissue in the body. Considering that Th2-type cytokines are generally associated with parasite persistence and growth $(14,15)$, a Th2-type immune response may facilitate the evasion of the host immune response by the parasite. High levels of cytokines, including IL-4, IL-5, IL-9 and IL-13, secreted by different types of immune cells in response to parasite antigens, not only reduce the Th1 response, but also promote parasite expulsion and tissue renewal and repair (16).

IL-17, induced by IL-23 and modulated by IL-21, IL-22 and TGF- $\beta 1$, is mainly involved in T-cell-mediated activation of innate immunity/inflammation, as well as immune tolerance (17). BALB/c mice infected with Schistosoma japonicum have been shown to exhibit significant changes in cytology, particularly the decrease in Th1 and plasma cells and increase in Th2 and Th17 cells (18). In the present study, levels of IL-17 were relatively low in the uninfected control; however, levels increased gradually following Em infection, particularly during the middle-late stage of infection. In the early stage of infection, Th1 and Th17 cells may have roles in clearing the parasites; however, with the extension of infection time, particularly after 3 months, Th2-type cytokines may begin to inhibit Th1-cell proliferation and immune response. Thus, Th1 cells were downregulated, and the high levels of IL-17 secreted by Th17 cells resulted in a strong immunopathological injury of the host liver. The Th2-type immune response can protect alveolar hydatids from the host immune attack. This may explain the slow growth of alveolar hydatids during the first 3 months post-infection and the rapid growth in the abdominal cavity and liver after 3 months, breaking through the diaphragmatic muscle and reaching the chest area.

Compared with the uninfected control, IL- 6 levels in the infected groups were increased and reached a maximum at 2 days post-infection. These data show that when the systemic immune response occurred following invasion of the body by alveolar hydatids, IL-6 participated in an acute inflammation process and levels of IL-6 increased rapidly at the early stage of infection. When Em parasites were unable to be cleared from the body, the inflammation continually persisted. The production of TGF- $\beta 1$ showed a similar trend to IL-17 and rapidly increased at the middle-late stage of infection. TGF- $\beta 1$ was highly expressed at the late stage of infection and exhibited similar variations in expression to IL-17, indicating that TGF- $\beta 1$ may have an important role in promoting Th17-cell differentiation. Hepatic stellate cells stimulated by TGF- $\beta 1$ can promote liver fibrosis (19). With the growth of hydatid cysts, inflammatory reaction infiltrates formed a fibrous layer and separated the hydatid cysts from the host tissue.

In conclusion, the present study may explain the cellular immunity observed in the Th1/Th2 axis and broaden the understanding of the immunopathological effects of Th17 cells in the development of AE.

\section{Acknowledgements}

The present study was supported by the Scientific Research Project of Science Department of Xinjiang Autonomous Region (no. 2012211A034) and the National Natural Science Foundation (nos. 31160194, 81260253, 30960358, 31000411, $30901374,81160378,81060135$ and 30860263) and the Scientific Research Project of the Science Department of Xinjiang Autonomous Region (no. 2012211A034).

\section{References}

1. Wang X, Lu R, Liu QF, et al: Production and immunoanalytical application of 32 monoclonal antibodies against metacestode somatic antigens of Echinococcus multilocularis. Parasitol Res 107: 177-185, 2010.

2. Barthelmann J, Nietsch J, Blessenohl M, et al: The protective Th1 response in mice is induced in the T-cell zone only three weeks after infection with Leishmania major and not during early T-cell activation. Med Microbiol Immunol 201: 25-35, 2012. 
3. Pang NN, Ding JB, Zhao H, et al: The dynamic observation of liver T-bet, GATA-3, ROR- $\gamma$ t and IL-17 mRNA expression in BALB/c mice infected with Echinococcus multilocularis. Chin J Immunol 27: 395-399, 2011 (In Chinese).

4. Ma XM, Xu Q, Hou M, et al: Effects of Echinococcus granulosus infection on IL-17 level and Th1/Th2 balance in serum of asthmatic rats. Immunol J 27: 110-113, 2011 (In Chinese).

5. Mendes EA, Mendes TA, dos Santos SL, et al: Expression of IL-4, IL-10 and IFN- $\gamma$ in the liver tissue of cattle that are naturally infected with Fasciola hepatica. Vet Parasitol 195 177-182, 2013.

6. Infante-Duarte C, Horton HF, Byrne MC and Kamradt T: Microbial lipopeptides induce the production of IL-17 in Th cells. J Immunol 165: 6107-6115, 2000.

7. Mbow M, Larkin BM, Meurs L, et al: T-helper 17 cells are associated with pathology in human schistosomiasis. J Infect Dis 207: 186-195, 2013.

8. Zhang Y, Chen L, Gao W, et al: IL-17 neutralization significantly ameliorates hepatic granulomatous inflammation and liver damage in Schistosoma japonicum infected mice. Eur J Immunol 42: 1523-1535, 2012.

9. Zhang H, Hu X, Liu X, et al: The Treg/Th17 imbalance in Toxoplasma gondii-infected pregnant mice. Am J Reprod Immunol 67: 112-121, 2012

10. Wang S, Xu Y, Zhu M, et al: Cytokine development in mice with secondary Echinococcus granulosus infection. Endemic Diseases Bulletin 17: 8-11, 2002 (In Chinese).
11. Wei XL, Ding JB, Xu Z, et al: Change of cytokines in mice with Echinococcus multilocularis infection. Chin J Parasitol Parasit Dis 22: 361-364, 2004 (In Chinese).

12. Rogan MT: T-cell activity associated with secondary infections and implanted cysts of Echinococcus granulosus in BALB/c mice. Parasite Immunol 20: 527-533, 1998.

13. Li Y, Terkawi MA, Nishikawa Y, et al: Macrophages are critical for cross-protective immunity conferred by Babesia microti against Babesia rodhaini infection in mice. Infect Immun 80: 311-320, 2012.

14. Xu X, Wen X, Chi Y, et al: Activation-induced T helper cell death contributes to Th1/Th2 polarization following murine Schistosoma japonicum infection. J Biomed Biotechnol 2010: 202397, 2010

15. Vuitton DA: The ambiguous role of immunity in echinococcosis: protection of the host or of the parasite? Acta Trop 85: 119-132, 2003.

16. Pennock JL and Grencis RK: The mast cell and gut nematodes: damage and defence. Chem Immunol Allergy 90: 128-140, 2006.

17. Wilson NJ, Boniface K, Chan JR, et al: Development, cytokine profile and function of human interleukin 17-producing helper T cells. Nat Immunol 8: 950-957, 2007.

18. Zhao JJ, Huang J, Mai JY, et al: Changes of cytology of spleen in BALB/c infected with Schistosoma japonicum. J Trop Med 9: 140-143, 2009 (In Chinese).

19. Jia D, Duan F, Peng P, et al: Up-regulation of RACK1 by TGF- $\beta 1$ promotes hepatic fibrosis in mice. PLoS One 8: e60115, 2013. 